Article

\title{
Impedance Modeling and Stability Analysis of VSG Controlled Grid-Connected Converters with Cascaded Inner Control Loop
}

\author{
Yunyang Xu, Heng Nian *, Yangming Wang and Dan Sun \\ College of Electrical Engineering, Zhejiang University, Hangzhou 310027, China; xuyunyang@zju.edu.cn (Y.X.); \\ wangyangming@zju.edu.cn (Y.W.); sundan@zju.edu.cn (D.S.) \\ * Correspondence: nianheng@zju.edu.cn
}

Received: 25 August 2020; Accepted: 29 September 2020; Published: 1 October 2020

check for updates

\begin{abstract}
This paper develops the impedance models of grid-connected converters under the virtual synchronous generator (VSG) strategy with a cascaded inner control loop and analyzes the system stability of VSG controlled converters with different kinds of weak grid. Different from existing small-signal models with high dimensions, a single-in-single-out (SISO) impedance model with simple mathematical expression is obtained in this paper, which is applied to identify the influence of the cascaded control loop on impedance characteristics and system stability. It is found that the impedance characteristics of VSG controlled converters can become capacitive below the fundamental frequency, and it is mainly caused by the voltage controller in the cascaded control loop of the VSG strategy. Impedance-based stability analysis shows that the capacitive impedance characteristics can benefit the compatibility of converters operated with the series-compensated weak grid, but may deteriorate the system stability with the inductive weak grid, which can be avoided by increasing the proportional coefficients of the cascaded voltage and current controllers or applying a larger virtual resistor to reduce the negative resistance in the capacitive frequency range. Experiments based on the control-hardware-in-loop (CHIL) platform were carried out to verify the developed analytical models and possible system instable cases.
\end{abstract}

Keywords: impedance model; small-signal modeling; system stability; virtual synchronous generator (VSG); weak grid

\section{Introduction}

With the development of renewable energy, the penetration of renewable energy generation systems (REGS) in the power system is gradually increasing. Most of the REGS connect to the grid through grid-connected converters and the grid-following control effects of the converters prevent REGS from providing inertial support to the power system. To address this problem, the concept of virtual synchronous generators (VSGs) is put forward, which emulates the behavior of traditional synchronous generators (SGs) and adds inertial to the power system by containing the mathematical model of a SG [1].

REGS usually connects to the weak grid with high impedance due to the long-distance transmission lines. In some places, series compensation devices are added to the weak grid to enhance transmission capability [2]. As reported in [3,4], stability accidents can be caused when the characteristics of the converter-based REGS do not match the connected weak grid. Thus, it is important to analyze the stability of VSG controlled converter system with the weak grid for practical applications.

The impedance-based stability analysis method is widely applied to analyze the stability of the grid-converter interconnected system [5,6]. In this method, the characteristics of the converter and 
the connected grid are described by their impedance models, respectively; the system stability can be analyzed with the impedance ratio of the converter and the connected grid; and the impedance ratio must satisfy the Nyquist stability criterion in order for the interconnected system to be stable [7].

The small-signal modeling and the impedance modeling of grid-connected converters under a typical VSG control strategy is analyzed in [8-14], where the output voltage reference from SG models is directly used to generate the PWM signals for converters as proposed in [15]. In [8], a line-frequency-averaged small-signal model is developed to design the power loop of the typical VSG strategy. In [9,10], small-signal state-space models are developed for VSG controlled converters with the connected grid, and critical parameters for system stability are identified with the eigenvalue analysis and the parameter sensitivity analysis. It is also found in [11-14] that the impedance characteristics of grid-connected converters under this typical VSG control strategy is generally inductive with relatively low magnitude, which is more compatible with the inductive weak grid than the traditional grid-following converter with a phase-locked loop (PLL), but grid-connected converters under this typical VSG control strategy also have the low-frequency oscillation risk with the series-compensated network similar as traditional SGs.

In practice, different implementations of VSG control strategy have been proposed [16-20]. In [18-20], a cascaded inner voltage-current control loop is adopted to regulate the output voltage reference from SG models. Virtual impedance control is also applied to emulate the quasi-stationary characteristics of the synchronous impedance in a traditional SG. Compared with above-mentioned typical VSG control strategy with direct open loop PWM control in [8-15], the cascaded inner control loop in the VSG strategy in [18-20] can conveniently implement the limitations and saturations of voltage and current outputs, which is a significant requirement to improve the current control capability and guarantee the safe operation of converters.

Recently, some small-signal modeling and analysis are carried to analyze the system stability of weak grid and the VSG controlled converters with cascaded inner control loop. In [18], a small-signal state-space model is developed to analyze the grid-converter interconnected system, and the system stability is assessed by eigenvalue analysis and the parametric sensitivities, while the impedance characteristics are not investigated. In [21], impedance models are transformed from the linearized state-space model, and the stability of this grid-converter interconnected system is mainly analyzed in the high frequency range (above $500 \mathrm{~Hz}$ ) where the control effects of the cascaded control loop is relatively limited. In [22], grid-connected converters under the power synchronization control and the vector control are compared with the developed $d q$-axis impedance models, and opposite characteristics of system stability are found between two control strategies.

However, it should be noted that the existing state-space model in [22] and the existing impedance models in [21,22] are all high-dimensional small-signal models, thus the characteristics of the VSG controlled converters can only be analyzed with a group of bode plots or with pole maps. Besides, the impedance model in [21] and the determinant of $d q$-axis impedance ratio matrix in [22] both lack mathematical expressions to insightfully analyze and quantify the impedance shaping effects of the cascaded control loop.

Therefore, an impedance model with simple mathematical expression is required and this paper will develop a novel single-in-single-out (SISO) impedance model with simple mathematical expression from the small-signal modeling of the VSG controlled converters with cascaded inner control loop. The applicable frequency range of the developed SISO impedance model is verified in the low frequency range $(1-100 \mathrm{~Hz})$, where the impedance characteristics are focused in this paper. The developed SISO impedance model is also applied to analyze the influence of the cascaded inner control loop on impedance characteristics and system stability.

The main contributions of this paper can be concluded as:

(1) A novel SISO impedance model with simple mathematical expression is developed to analyze the impedance characteristics of the VSG controlled grid-connected converters with the cascaded inner control loop. 
(2) Based on the developed SISO impedance model, the analysis on how the impedance characteristics are shaped by controllers in the cascaded inner control loop is provided.

(3) Impedance-based system stability of VSG controlled converters with different kinds of weak grid is analyzed, and the influence of cascaded inner control loop on possible system instabilities are quantified by differentiating the expression of developed SISO impedance model.

The rest of this paper is organized as follows. In Section 2, detailed small-signal modeling of the VSG controlled converters with cascaded inner control loop is carried out. Section 3 obtains the SISO impedance models from the developed small-signal models and analyzes the impedance characteristics of VSG controlled converters. Section 4 identifies the influence of cascaded inner control loop on impedance characteristics and quantifies the influence of the control parameters. In Section 5, the impedance-based analysis is carried out to analyze the system stability of VSG controlled converters with different kinds of weak grid, based on which possible causes of system instability are also identified. Experiments based on the control-hardware-in-loop (CHIL) platform are presented to validate the developed models and the possible system instable cases. Finally, Section 6 concludes this work.

\section{Small-Signal Modeling of VSG Controlled Converters with Cascaded Inner Control Loop}

In this section, detailed small-signal modeling of the VSG controlled converters is carried out by applying the multi-harmonic linearization method as in [23-26]. The small-signal characteristics are described by a developed $2 \times 2$ order admittance matrix model, and CHIL-based frequency sweeping test is applied to validate the correctness of the developed small-signal models.

\subsection{VSG Control Strategy with Cascaded Inner Control Loop}

In this paper, the VSG strategy as in [18] is applied. In this VSG strategy, the inertial and damping characteristics of traditional SGs are implemented with a swing equation as:

$$
\frac{d \omega_{p u}}{d t}=\frac{P_{r e f}}{J}-\frac{P}{J}-\frac{D_{p}\left(\omega_{p u}-\omega_{g, p u}\right)}{J}
$$

In (1), the per unit angular speed $\omega_{p u}$ is determined by the integral of the active power balance between the active power reference $P_{\text {ref }}$ and the measured active power $P$. The damping characteristics of traditional SGs are emulated by a damping active power, which is defined by the damping coefficient $D_{p}$ with the difference between the per unit angular speed $\omega_{p u}$ and the per unit angular frequency of the connected grid $\omega_{g, p u}$. For VSG controlled converters, the per unit angular speed $\omega_{p u}$ equals to the grid frequency $\omega_{g, p u}$ in a stable grid-connected operating state, and $\omega_{g, p u}$ of a normal power grid equals 1 in steady operating state.

Then, the virtual rotor angular position $\theta$ can be calculated by the integral of the per unit angular speed $\omega_{p u}$ with the base of angular speed $\omega_{\text {base }}$ as:

$$
\frac{d \theta}{d t}=\omega_{p u} \cdot \omega_{\text {base }}
$$

In this applied VSG strategy, the virtual back electrical magnetic field (EMF) $\psi_{f}$ is regulated by reactive power control as:

$$
\frac{d \psi_{f}}{d t}=K \cdot\left(Q_{r e f}-Q+Q_{d}\right), Q_{d}=D_{q}\left(\left|V_{r e f}\right|-|V|\right)
$$

where the virtual EMF is determined by the integral of the reactive power balance among reactive power reference $Q_{r e f}$, the measured reactive power $Q$, and the damping reactive power $Q_{d}$. The damping reactive power $Q_{d}$ is defined by the damping coefficient $D_{q}$ and the difference between the voltage 
magnitude reference $\left|V_{\text {ref }}\right|$ and the measured voltage magnitude of the converter. In steady operating state, $\left|V_{\text {ref }}\right|$ is set to 1.0.

When adopting a cascaded inner control loop with virtual impedance in the VSG strategy, the $d q$-axis voltage references for modulations are selected as the output references of the cascaded control loop, otherwise the virtual EMF is directly selected as $d$-axis voltage reference.

In this paper, the configuration and applied VSG control strategy of the grid-connected converter system are shown in Figure 1, where the switch of modulation references turns to the upper side to select the output references of the cascaded control with the adoption of cascaded control.

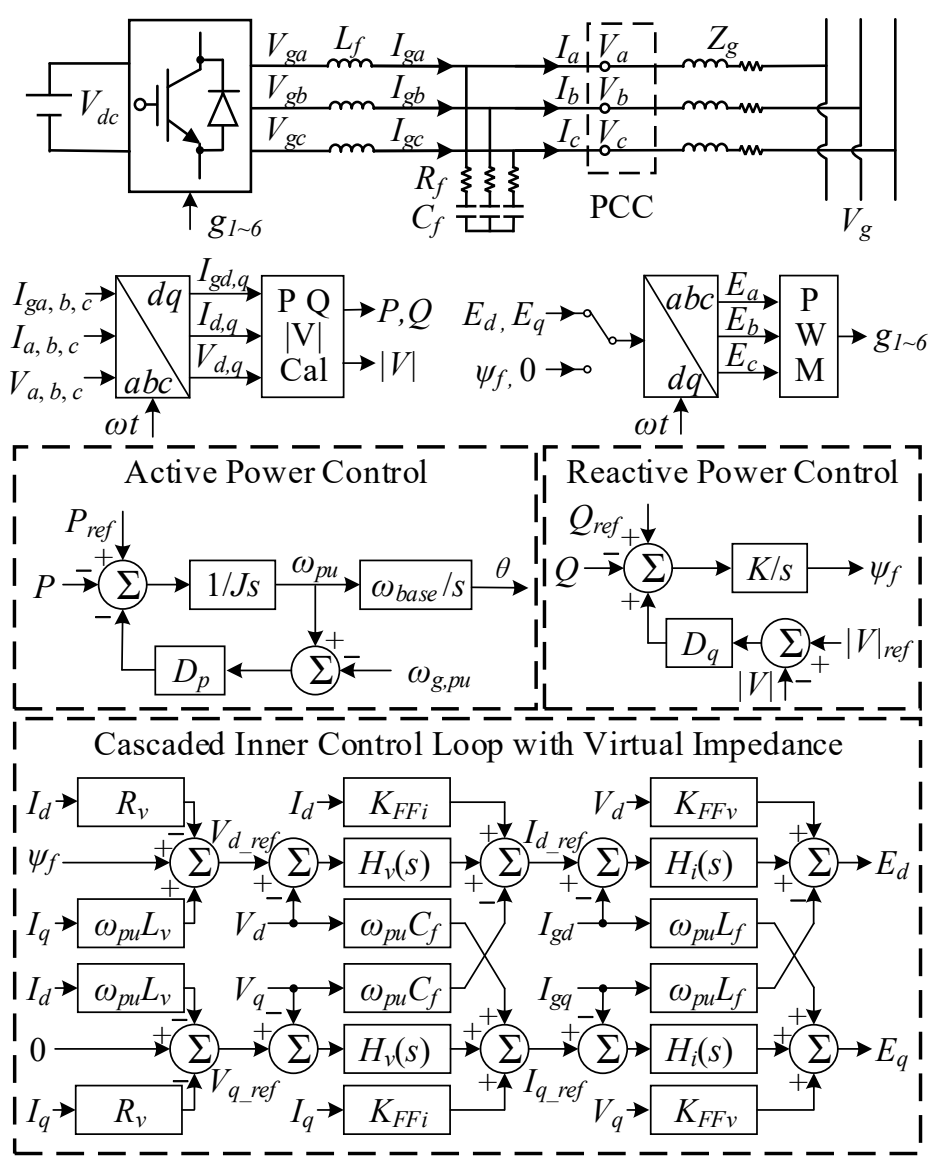

Figure 1. Configuration of VSG controlled converter system.

\subsection{Definition of Small-Signal Models}

In small-signal modeling, the characteristics of grid-connected converters can be described by the relationship between the ac-terminal voltage small-signal perturbations and corresponding current responses. Since the frequency coupling effect, as analyzed in [23-27], exists in converters, the component at a given frequency $f_{p}$ and another component at the frequency $f_{p}-2 f_{1}$ always coexist in small-signal modeling, where $f_{1}$ is the fundamental frequency of the system.

Following the same notation as in [23-25], in the frequency domain, the perturbed components of system voltages and currents can be defined as:

$$
\hat{i}=\left[\begin{array}{ll}
\hat{\mathbf{I}}_{p} & \hat{\mathbf{I}}_{p 2}
\end{array}\right]^{T}, \hat{v}=\left[\begin{array}{ll}
\hat{\mathbf{V}}_{p} & \hat{\mathbf{V}}_{p 2}
\end{array}\right]^{T}
$$

where $\hat{\mathbf{I}}_{p}, \hat{\mathbf{V}}_{p}, \hat{\mathbf{I}}_{p 2}$, and $\hat{\mathbf{V}}_{p 2}$ are components at frequency $f_{p}$ and frequency $f_{p}-2 f_{1}$, respectively, and the symbol "^" means perturbing components. 
Then, the small-signal models of VSG controlled converters can be described by a $2 \times 2$ order admittance matrix $\mathbf{Y}_{V S G}$ as in [23-25], and the degree of frequency coupling effect is represented by the magnitude ratio of the off-diagonal elements to the diagonal elements.

$$
\left[\begin{array}{c}
\hat{\mathbf{I}}_{p} \\
\hat{\mathbf{I}}_{p 2}
\end{array}\right]=-\underbrace{\left[\begin{array}{ll}
Y_{11}(s) & Y_{12}(s) \\
Y_{21}(s) & Y_{22}(s)
\end{array}\right]}_{\mathbf{Y}_{V S G}}\left[\begin{array}{c}
\hat{\mathbf{V}}_{p} \\
\hat{\mathbf{V}}_{p 2}
\end{array}\right]
$$

\subsection{Small-Signal Modeling of VSG Control Strategy}

\subsubsection{Modeling of Power Relationship}

In converter system, active and reactive power can be calculated with voltages and currents as:

$$
\begin{gathered}
P(t)=v_{a}(t) \cdot i_{a}(t)+v_{b}(t) \cdot i_{b}(t)+v_{c}(t) \cdot i_{c}(t) \\
Q(t)=-\frac{1}{\sqrt{3}}\left(v_{b c}(t) \cdot i_{a}(t)+v_{c a}(t) \cdot i_{b}(t)+v_{a b}(t) \cdot i_{c}(t)\right)
\end{gathered}
$$

In small-signal modeling, the active and reactive power fluctuations can be expressed with the voltage perturbations and current responses by linearizing (6) and (7) as:

$$
\begin{aligned}
& {[\hat{\mathbf{P}}]=\underbrace{\left[\begin{array}{ll}
\mathbf{I}_{1}^{*} & \mathbf{I}_{1}
\end{array}\right]}_{G_{v 2 p}} \cdot\left[\begin{array}{c}
\hat{\mathbf{V}}_{p} \\
\hat{\mathbf{V}}_{p 2}
\end{array}\right]+\underbrace{\left[\begin{array}{ll}
\mathbf{V}_{1}^{*} & \mathbf{V}_{1}
\end{array}\right]}_{G_{i 2 p}} \cdot\left[\begin{array}{c}
\hat{\mathbf{I}}_{p} \\
\hat{\mathbf{I}}_{p 2}
\end{array}\right]} \\
& {[\hat{\mathbf{Q}}]=\underbrace{\left[\begin{array}{ll}
-j \mathbf{I}_{1}^{*} & j \mathbf{I}_{1}
\end{array}\right]}_{G_{v 2 q}} \cdot\left[\begin{array}{c}
\hat{\mathbf{V}}_{p} \\
\hat{\mathbf{V}}_{p 2}
\end{array}\right]+\underbrace{\left[\begin{array}{ll}
j \mathbf{V}_{1}^{*}-j \mathbf{V}_{1}
\end{array}\right]}_{G_{i 2 q}} \cdot\left[\begin{array}{c}
\hat{\mathbf{I}}_{p} \\
\mathbf{I}_{p 2}
\end{array}\right]}
\end{aligned}
$$

where $\hat{\mathbf{P}}$ and $\hat{\mathbf{Q}}$ are perturbed power components at frequency $f_{p}-f_{1} ; \mathbf{I}_{1}$ and $\mathbf{V}_{1}$ are fundamental components of PCC current and voltage in p.u.; * means conjugate operation; and $G_{v 2 p}, G_{v 2 q}, G_{i 2 p}$, and $G_{i 2 q}$ describe the relationship between power fluctuations and voltage/current perturbations.

\subsubsection{Modeling of Active and Reactive Power Control}

According to Figure 1, the virtual rotor angular position is affected by active power fluctuations and can be written as:

$$
[\hat{\theta}]=G_{p 2 \theta} \cdot[\hat{\mathbf{P}}], G_{p 2 \theta}=-\frac{\omega_{\text {base }} / s}{J s+D_{p}}
$$

where $s=j 2 \pi\left(f_{p}-f_{1}\right), \omega_{\text {base }}=2 \pi f_{1}$, and $\hat{\theta}$ is the perturbed component of virtual rotor angular position at frequency $f_{p}-f_{1}$.

According to the reactive power control in Figure 1, the perturbed virtual back EMF $\psi_{f}$ can be expressed with the reactive power and voltage fluctuations as:

$$
\begin{gathered}
{[\hat{\boldsymbol{\Psi}}]=G_{q 2 \psi} \cdot\left(\hat{\mathbf{Q}}+G_{m 2 q} \cdot|\hat{V}|\right), G_{q 2 \psi}=-K / s, G_{m 2 q}=D_{q}} \\
|\hat{V}|=G_{v 2 m} \cdot\left[\begin{array}{ll}
\hat{\mathbf{V}}_{p} & \hat{\mathbf{V}}_{p 2}
\end{array}\right]^{T}, G_{v 2 m}=\left[\begin{array}{ll}
\mathbf{V}_{1}^{*} & \mathbf{V}_{1}
\end{array}\right]
\end{gathered}
$$


where $s=j 2 \pi\left(f_{p}-f_{1}\right), \hat{\psi}$ is the perturbed component of $\psi_{f}$ at frequency $f_{p}-f_{1}, K$ is the coefficient of the integral controller, and $D_{q}$ is the coefficient of voltage droop controller.

\subsubsection{Modeling of Cascaded Inner Control Loop with Virtual Impedance}

According to Figure 1, in small-signal modeling, the perturbed $d q$-axis output voltage references can be expressed with perturbed virtual EMF output, perturbed $d q$-axis PCC voltages, perturbed $d q$-axis PCC currents, and perturbed $d q$-axis output currents of the converter as:

$$
\left[\begin{array}{l}
\hat{\mathbf{E}}_{d} \\
\hat{\mathbf{E}}_{q}
\end{array}\right]=\mathbf{T}_{d q, \psi}[\hat{\boldsymbol{\Psi}}]+\mathbf{T}_{d q, v}\left[\begin{array}{l}
\hat{\mathbf{V}}_{d} \\
\hat{\mathbf{V}}_{q}
\end{array}\right]+\mathbf{T}_{d q, i}\left[\begin{array}{l}
\hat{\mathbf{I}}_{d} \\
\hat{\mathbf{I}}_{q}
\end{array}\right]+\mathbf{T}_{d q, i g}\left[\begin{array}{l}
\hat{\mathbf{I}}_{q d} \\
\hat{\mathbf{I}}_{g q}
\end{array}\right]
$$

where $s=j 2 \pi\left(f_{p}-f_{1}\right) ; \hat{\mathbf{E}}_{d}, \hat{\mathbf{E}}_{q}, \hat{\mathbf{V}}_{d}, \hat{\mathbf{V}}_{q}, \hat{\mathbf{I}}_{d}, \hat{\mathbf{I}}_{q}, \hat{\mathbf{I}}_{q d}$, and $\hat{\mathbf{I}}_{g q}$ are components at frequency $f_{p}-f_{1} ;$ and matrices $\mathbf{T}_{d q, \psi}, \mathbf{T}_{d q, v}, \mathbf{T}_{d q, I}$, and $\mathbf{T}_{d q, i g}$ are defined for abbreviation as:

$$
\begin{aligned}
& \mathbf{T}_{d q, \psi}=\left[\begin{array}{c}
H_{v}(s) H_{i}(s) \\
0
\end{array}\right], \mathbf{T}_{d q, v}=-\left[\begin{array}{cc}
H_{v}(s) H_{i}(s)-K_{F F v} & C_{f} H_{i}(s) \\
-C_{f} H_{i}(s) & H_{v}(s) H_{i}(s)-K_{F F v}
\end{array}\right], \\
& \mathbf{T}_{d q, i}=-\left[\begin{array}{cc}
R_{v} H_{v}(s) H_{i}(s) & -L_{v} H_{v}(s) H_{i}(s) \\
L_{v} H_{v}(s) H_{i}(s) & R_{v} H_{v}(s) H_{i}(s)
\end{array}\right], \mathbf{T}_{d q, i g}=-\left[\begin{array}{cc}
H_{i}(s) & L_{f} \\
-L_{f} & H_{i}(s)
\end{array}\right]
\end{aligned}
$$

Since the cascaded control is carried out in $d q$-frame, the perturbed components of the virtual rotor angular position will also affect the $d q$-axis output voltage references. The perturbed $d q$-axis voltages and currents satisfy:

$$
\begin{gathered}
{\left[\begin{array}{ll}
\hat{\mathbf{V}}_{d} & \hat{\mathbf{V}}_{q}
\end{array}\right]^{T}=\mathbf{T}_{p n 2 d q}\left[\begin{array}{ll}
\hat{\mathbf{V}}_{p} & \hat{\mathbf{V}}_{p 2}
\end{array}\right]^{T}+\mathbf{T}_{\theta, v}[\hat{\theta}]} \\
{\left[\begin{array}{ll}
\hat{\mathbf{I}}_{d} & \hat{\mathbf{I}}_{q}
\end{array}\right]=\mathbf{T}_{p n 2 d q}\left[\begin{array}{ll}
\hat{\mathbf{I}_{p}} & \hat{\mathbf{I}}_{p 2}
\end{array}\right]+\mathbf{T}_{\theta, i}[\hat{\theta}]} \\
{\left[\begin{array}{ll}
\hat{\mathbf{I}}_{g d} & \hat{\mathbf{I}}_{g q}
\end{array}\right]=\mathbf{T}_{p n 2 d q}\left[\begin{array}{ll}
\hat{\mathbf{I}}_{g p} & \hat{\mathbf{I}}_{g p 2}
\end{array}\right]+\mathbf{T}_{\theta, i g}[\hat{\theta}}
\end{gathered}
$$

where $\theta_{0}, \varphi_{v 1}, \varphi_{i 1}$, and $\varphi_{i g 1}$ are the initial angle of the virtual rotor angular position, PCC voltage, PCC current, and output current of the converter, respectively, and matrices $\mathbf{T}_{p n 2 d q}$ and $\mathbf{T}_{\theta, x}\left(x=v, i, i_{g}\right)$ are defined for abbreviation as:

$$
\mathbf{T}_{p n 2 d q}=\left[\begin{array}{cc}
e^{-j \theta_{0}} & e^{j \theta_{0}} \\
-j e^{-j \theta_{0}} & j e^{j \theta_{0}}
\end{array}\right], \mathbf{T}_{\theta, x}=\left[\begin{array}{c}
X_{1, p u} \sin \left(\varphi_{x 1}-\theta_{0}\right) \\
-X_{1, p u} \cos \left(\varphi_{x 1}-\theta_{0}\right)
\end{array}\right]
$$

According to the LC filter structure of the converter, the relationship between the perturbed output current and PCC voltage and current can be represented as:

$$
\left[\begin{array}{ll}
\hat{\mathbf{I}}_{g p} & \hat{\mathbf{I}}_{g p 2}
\end{array}\right]^{T}=\left[\begin{array}{ll}
\hat{\mathbf{I}}_{p} & \hat{\mathbf{I}}_{p 2}
\end{array}\right]^{T}+\mathbf{Y}_{c f}\left[\begin{array}{ll}
\hat{\mathbf{V}}_{p} & \hat{\mathbf{V}}_{p 2}
\end{array}\right]^{T}
$$

where $s=j 2 \pi f_{p}$ and matrix $\mathbf{Y}_{c f}$ is the admittance of the filter resistor $R_{f}$ and filter capacitor $C_{f}$ as:

$$
\mathbf{Y}_{c f}=\operatorname{diag}\left\{\frac{1}{s C_{f}}+R_{f}, \frac{1}{\left(s-2 j \omega_{1}\right) C_{f}}+R_{f}\right\}^{-1}
$$

Then, by substituting (14)-(19) into (12), the perturbed $d q$-axis output voltage references of the cascaded control loop satisfy: 


$$
\left[\begin{array}{l}
\hat{\mathbf{E}}_{d} \\
\hat{\mathbf{E}}_{q}
\end{array}\right]=G_{\psi 2 E}[\hat{\boldsymbol{\psi}}]+G_{v 2 E}\left[\begin{array}{c}
\hat{\mathbf{V}}_{p} \\
\hat{\mathbf{V}}_{p 2}
\end{array}\right]+G_{i 2 E}\left[\begin{array}{c}
\hat{\mathbf{I}}_{p} \\
\hat{\mathbf{I}}_{p 2}
\end{array}\right]+G_{\theta 2 E}[\hat{\boldsymbol{\theta}}]
$$

where detailed expressions of $G_{\psi 2 E}, G_{v 2 E}, G_{i 2 E}$, and $G_{\theta 2 E}$ are as:

$$
\begin{gathered}
G_{\psi 2 E}=\left[\begin{array}{ll}
H_{v}(s) H_{i}(s) & 0
\end{array}\right]^{T} \\
G_{v 2 E}=\mathbf{T}_{d q, v} \cdot \mathbf{T}_{p n 2 d q}+\mathbf{T}_{d q, i g} \cdot \mathbf{T}_{p n 2 d q} \cdot \mathbf{Y}_{c f} \\
G_{i 2 E}=\mathbf{T}_{d q, i} \cdot \mathbf{T}_{p n 2 d q}+\mathbf{T}_{d q, i g} \cdot \mathbf{T}_{p n 2 d q} \\
G_{\theta 2 E}=\mathbf{T}_{d q, v} \cdot \mathbf{T}_{\theta, v}+\mathbf{T}_{d q, i} \cdot \mathbf{T}_{\theta, i}+\mathbf{T}_{d q, i g} \cdot \mathbf{T}_{\theta, i g}
\end{gathered}
$$

\subsubsection{Modeling of Modulation}

According to Figure 1, the output current of the converter and the terminal voltage of converter in $\mathrm{a} / \mathrm{b} / \mathrm{c}-$ phase can be expressed as:

$$
\begin{gathered}
L_{f} \frac{d i_{g x}(t)}{d t}=V_{g x}(t)-V_{x}(t) \\
V_{g x}(t)=K_{m} V_{d c} \cdot E_{x}(t)
\end{gathered}
$$

where $x=a, b, c$ indicating that, for variables in a/b/c-phase, $K_{m}$ is the modulating coefficient and $V_{d c}$ is the dc-link voltage.

In small-signal modeling, the perturbed voltage references in sequence domain can be transformed from $d q$-axis as:

$$
\left[\begin{array}{c}
\hat{\mathbf{E}}_{p} \\
\hat{\mathbf{E}}_{p 2}
\end{array}\right]=\left(\mathbf{T}_{p n 2 d q}\right)^{-1}\left\{\left[\begin{array}{l}
\hat{\mathbf{E}}_{d} \\
\hat{\mathbf{E}}_{q}
\end{array}\right]+\left[\begin{array}{c}
E_{1} \sin \left(\varphi_{e 1}-\theta_{0}\right) \\
-E_{1} \cos \left(\varphi_{e 1}-\theta_{0}\right)
\end{array}\right][\hat{\theta}]\right\}
$$

where $\varphi_{e 1}, \hat{\mathbf{E}}_{p}$, and $\hat{\mathbf{E}}_{p 2}$ are the initial angle and the components at frequency $f_{p}$ and $f_{p}-2 f_{1}$ of the output voltage references from the cascaded inner control loop, respectively.

By substituting (27) into (25) and (26), and considering the filter admittance in (19), the perturbed current can be described with voltage perturbations, the perturbed voltage references, and the perturbed virtual angular position as:

$$
\begin{aligned}
& {\left[\begin{array}{l}
\hat{\mathbf{I}}_{p} \\
\hat{\mathbf{I}}_{p 2}
\end{array}\right]=G_{v 2 i}\left[\begin{array}{c}
\hat{\mathbf{V}}_{p} \\
\hat{\mathbf{V}}_{p 2}
\end{array}\right]+G_{E 2 i}\left[\begin{array}{c}
\hat{\mathbf{E}}_{d} \\
\hat{\mathbf{E}}_{q}
\end{array}\right]+G_{\theta 2 i}[\hat{\theta}]} \\
& G_{v 2 i}=\operatorname{diag}\left\{-\frac{1}{s L_{f}}, \quad-\frac{1}{\left(s-j 2 \omega_{1}\right) L_{f}}\right\}-\mathbf{Y}_{c f} \\
& G_{E 2 i}=\operatorname{diag}\left\{\begin{array}{ll}
\frac{K_{m}(s) V_{d c}}{s L_{f}} & \frac{K_{m}\left(s-j 2 \omega_{1}\right) V_{d c}}{\left(s-j 2 \omega_{1}\right) L_{f}}
\end{array}\right\}\left(\mathbf{T}_{p n 2 d q}\right)^{-1} \\
& G_{\theta 2 i}=\left[\begin{array}{ll}
\frac{j}{2} \frac{K_{m}(s) V_{d c} \cdot \mathbf{E}_{1}}{s L_{f}} & -\frac{j}{2} \frac{K_{m}\left(s-j 2 \omega_{1}\right) V_{d c} \cdot \mathbf{E}_{1}{ }^{*}}{\left(s-j 2 \omega_{1}\right) L_{f}}
\end{array}\right]^{T}
\end{aligned}
$$

where $s=j 2 \pi f_{p}, \omega_{1}=2 \pi f_{1}$, and $\mathbf{E}_{1}$ is the fundamental component of the output voltage reference; the sampling and control delay is modeled in the transfer function $K_{m}(s)$.

\subsection{Small-Signal Models of VSG Controlled Converter System}

The block diagram shown in Figure 2 can be used to describe the small-signal relationship in the VSG controlled converter system, where blocks in four different colors correspond to the above modeling of power relationship, power control, cascaded inner control loop, and modulation, respectively. 


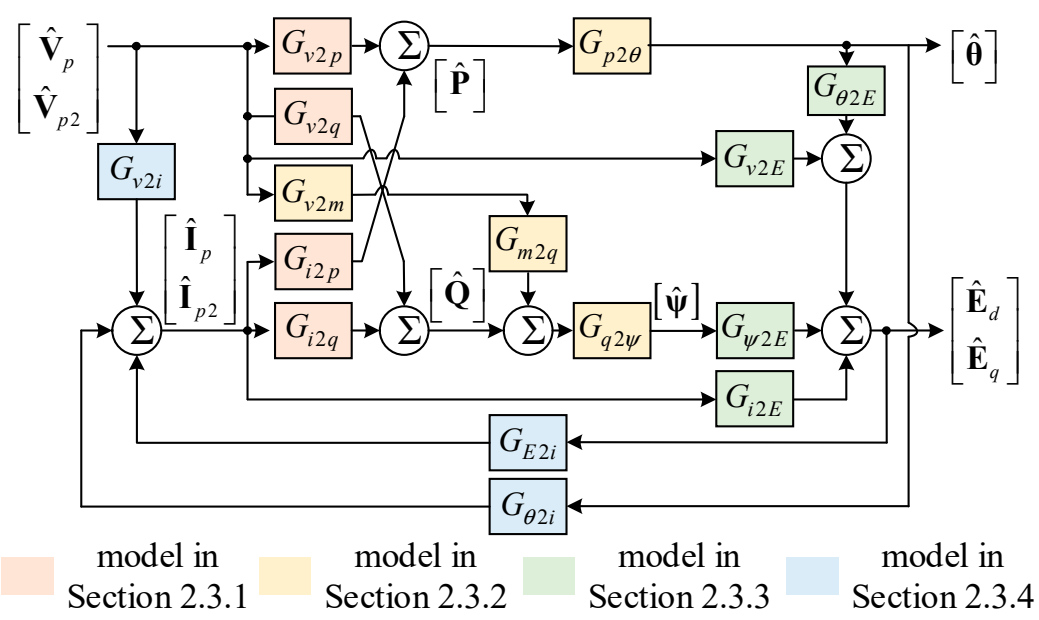

Figure 2. Small-signal relationship in the VSG controlled converter system.

Then, based on the small-signal relationship in Figure 2 and the model definition in (2), the small-signal models of the VSG controlled converter can be obtained as:

$$
\mathbf{Y}_{V S G}=\left(\mathbf{I}-\mathbf{M}_{i}\right)^{-1} \cdot \mathbf{M}_{v}
$$

where $\mathbf{I}$ is a unit matrix and $\mathbf{M}_{i}$ and $\mathbf{M}_{v}$ are abbreviated as:

$$
\begin{gathered}
\mathbf{M}_{i}=G_{\theta 2 i} G_{p 2 \theta} G_{i 2 p}+G_{E 2 i}\left(G_{i 2 E}+G_{\theta 2 E} G_{p 2 \theta} G_{i 2 p}+G_{\psi 2 E} G_{q 2 \psi} G_{i 2 q}\right) \\
\mathbf{M}_{v}=G_{v 2 i}+G_{\theta 2 i} G_{p 2 \theta} G_{v 2 p}+G_{E 2 i}\left(G_{v 2 E}+G_{\theta 2 E} G_{p 2 \theta} G_{v 2 p}+G_{\psi 2 E} G_{q 2 \psi}\left(G_{v 2 q}+G_{m 2 q} G_{v 2 m}\right)\right)
\end{gathered}
$$

To validate the correctness of the developed small-signal models in (32), frequency sweeping experiments based on CHIL platform were applied to obtain the measured small-signal models as in [23-25]. Detailed introductions about the frequency sweeping test are given in [28], and the picture of the CHIL platform is given in Figure 3. The main circuit of the grid-connected converter system was emulated in the Typhoon HIL 602+ platform with the time step of $1 \mu \mathrm{s}$. Controllers were implemented on a TMS320F28335 DSP+FPGA control board and the sampling and switching frequency was set as $5 \mathrm{kHz}$. System and control parameters of the VSG controlled converter system are listed in Table 1. The control bandwidths of active power controller, reactive power controller, the cascaded voltage controller, and current controller were $1.7,2.2,54$, and $155 \mathrm{~Hz}$, respectively.

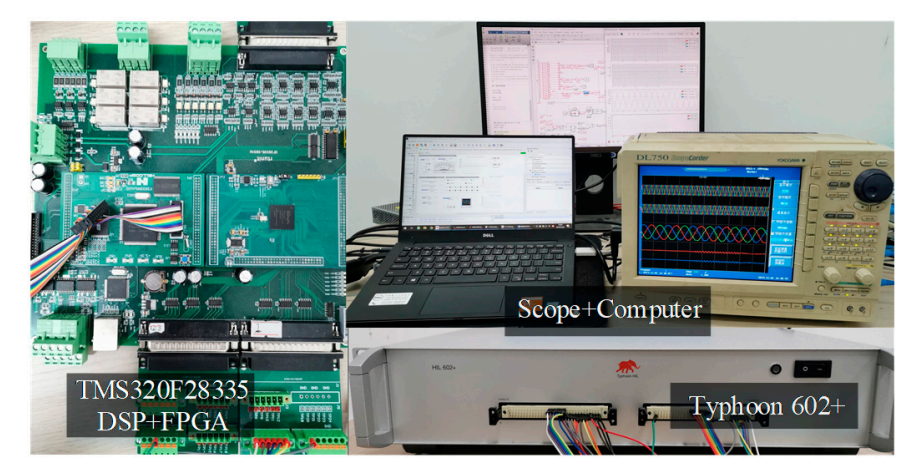

Figure 3. CHIL platform used in the following experiments. 
Table 1. Parameters of VSG controlled converter system.

\begin{tabular}{cccc}
\hline Parameter & Value & Parameter & Value \\
\hline Rated voltage, $U_{s}$ & $690 \mathrm{~V}$ & Damping coefficient, $D_{p}$ & 400 \\
Rated power, $S_{b}$ & $2.75 \mathrm{MW}$ & Voltage reference, $|\mathrm{V}|_{\text {ref }}$ & $1.0 \mathrm{p} . \mathrm{u}$. \\
Rated frequency, $f_{1}$ & $50 \mathrm{~Hz}$ & Reactive power reference, $Q_{\text {ref }}$ & $0.0 \mathrm{p} . \mathrm{u}$. \\
Power reference, $P_{r e f}$ & $0.5 \mathrm{p} . \mathrm{u}$. & Voltage droop coefficient, $D_{q}$ & 0.02 \\
Inertial constant, $J$ & $2 \mathrm{~s}$ & Integral coefficient, $K$ & 0.5 \\
Filter resistor, $R_{f}$ & 0.003 p.u. & Voltage controller, $k_{v p}, k_{v i}$ & 0.59736 \\
Filter inductor, $L_{f}$ & 0.08 p.u. & Current controller, $k_{i p}, k_{i i}$ & 1.2714 .3 \\
Filter capacitor, $C_{f}$ & 0.074 p.u. & Virtual impedance, $L_{v}, R_{v}$ & 0.20 .01 \\
\hline
\end{tabular}

The measurements from the frequency sweeping test and analytical small-signal models $\mathbf{Y}_{V S G}$ in (32) are plotted in Figure 4. From the results in Figure 4, the following conclusions can be obtained:

(1) The analytical small-signal models $\mathbf{Y}_{V S G}$ in (32) are in accordance with the measured results, which proves that the developed small-signal models of VSG controlled converters with cascaded inner control loop are correct.

(2) The magnitude of off-diagonal elements in the developed admittance matrix $\mathbf{Y}_{V S G}$ is lower than the diagonal elements except around the fundamental frequency, which means the frequency coupling effect can be ignored except near the fundamental frequency.
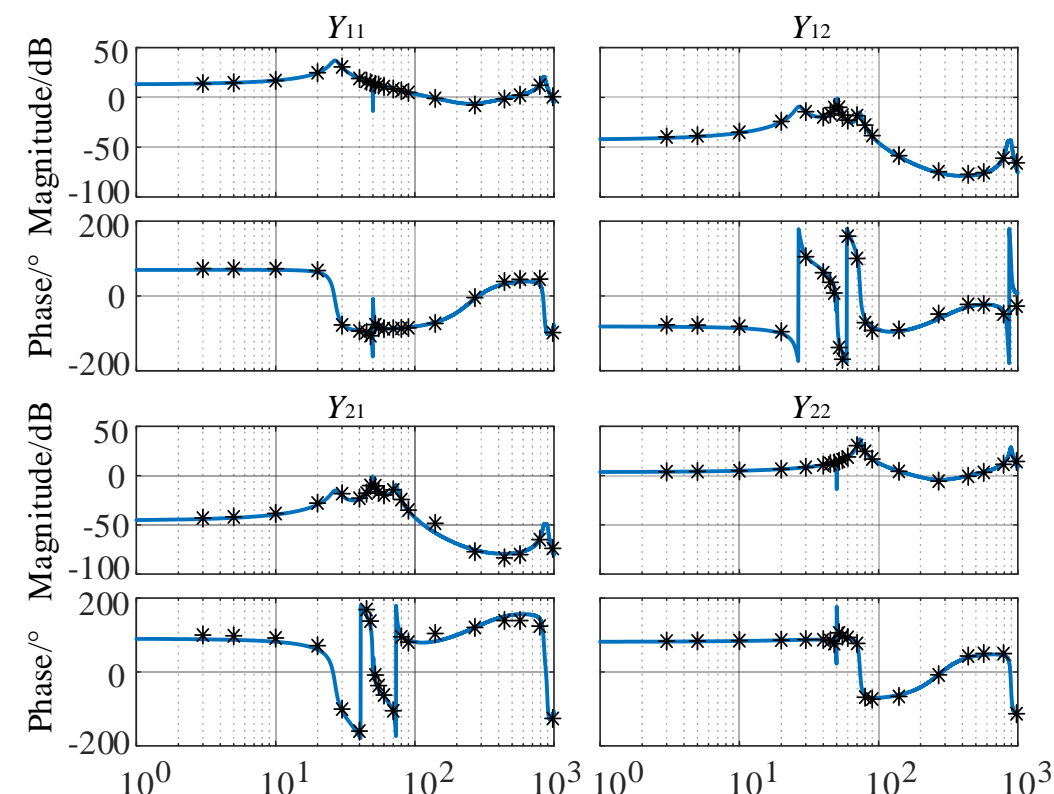

Frequency/Hz

- small-signal models $\mathbf{Y}_{V S G} \quad *$ measurements from frequency sweeping test

Figure 4. Validation of small-signal models $\mathbf{Y}_{V S G}$.

\section{Impedance Models and Impedance Characteristics of VSG Controlled Converter System}

In this section, a SISO impedance model with simple mathematical expressions is simplified from above small-signal models in (32), based on which the impedance characteristics of the VSG controlled converter system with the cascaded inner control loop are analyzed.

\subsection{A Simplified SISO Impedance Model}

When focusing on the impedance shaping effects of cascaded inner control loop, the following simplifications can be applied to the developed small-signal models $\mathbf{Y}_{V S G}$ in (32) to obtain a simpler expression to benefit following impedance analysis. 
Firstly, since the control effects of the cascaded voltage-current controller are relatively limited outside their control bandwidths, the sampling and control delay modeled by $K_{m}(s)$ can be ignored below $500 \mathrm{~Hz}$ (1/10 of the switching frequency $5 \mathrm{kHz})$. The resonance frequency of the filter inductor and filter capacitor is about $650 \mathrm{~Hz}$; thus, the influence of delay and filter capacitor are seen as the characteristics in the high frequency range (above $500 \mathrm{~Hz}$ ), and will not be concerned in the following analysis. Then, under this assumption, $K_{m}(s)$ equals constant gain $K_{m}$ and satisfies $K_{m}{ }^{*} V_{d c}=1$, while the admittance of the filter resistor and filter capacitor in (19) satisfies $\mathbf{Y}_{c f}=0$.

Secondly, the frequency coupling effects caused by the active and reactive power control are ignored since the control bandwidth of power controller $(1-2 \mathrm{~Hz})$ is much smaller than the cascaded voltage and current controller $(50-200 \mathrm{~Hz})$. Then, under this assumption, the positive-sequence admittance of VSG controlled converters in (32) can be obtained by calculating $\mathbf{M}_{v}(1,1) /\left(1-\mathbf{M}_{i}(1,1)\right)$, where $(1,1)$ indicates the first row and first line elements in the matrices.

Finally, setting the feedforward gain $K_{F F v}=1$ and $K_{F F i}=0$, simplifying the expressions according to the control bandwidths of different controllers, and substituting the detailed expressions of $G_{p 2 \theta}$ in (9) and $G_{q 2 \psi}$ in (10), a positive-sequence SISO impedance model can be simplified from (32) as (35). The Appendix A shows the detailed explanation and derivation of the simplified SISO impedance model.

$$
Z_{p}^{v s g} \approx Z_{v}+\frac{1}{H_{v}(s)}+\frac{\left(s+j 2 \pi f_{1}\right) L_{f}}{H_{v}(s) \cdot H_{i}(s)}-\frac{j}{2} \cdot \frac{\omega_{\text {base }}}{J s^{2}+D_{p} s}-\frac{j}{2} \cdot \frac{K}{s}
$$

where $s=j 2 \pi\left(f_{p}-f_{1}\right)$ and $Z_{v}=R_{v}+j L_{v}$.

Different from existing impedance models with high dimensions in [21,22], the impedance model in (35) is a SISO model with simple mathematical expressions. Thus, the impedance characteristics of the VSG controlled converters with the cascaded inner control loop can be presented with a bode plot of (35), rather than a group of bode plots with pole maps as in [21,22]. The mathematical expression of the SISO impedance model in (35) can also be applied to indicate how each controller shapes the impedance characteristics of the VSG controlled converters and quantify how the impedance characteristics are influenced by the control parameters in the following impedance analysis.

\subsection{Validation of Simplified SISO Impedance Model}

Before identifying the impedance shaping effects of the cascaded inner control loop, the correctness of the simplified SISO impedance model in (35) should be validated to define the frequency range where the SISO impedance model applies, and the small-signal models $\mathbf{Y}_{V S G}$ in (32) are used as references.

Since the small-signal models in (32) is a $2 \times 2$ order admittance matrix, the following conversion, as adopted in [25], is applied to (32):

$$
Y_{p}^{q q l}=Y_{11}-\frac{Y_{21} \cdot Y_{12} \cdot Z_{g p 2}}{1+Y_{22} \cdot Z_{g p 2}}, Z_{p}^{q q l}=\frac{1}{Y_{p}^{q q l}}
$$

where $Y_{p}^{e q l}$ and $Z_{p}^{e q l}$ are equivalent admittance and impedance model of the small-signal models $\mathbf{Y}_{V S G}$ in (32) and $Z_{g p}$ and $Z_{g p 2}$ are defined as the impedances of ac-grid at frequency $f_{p}$ and $f_{p}-2 f_{1}$, respectively.

If the cascaded inner control loop is not adopted in the VSG control strategy, the switch of modulation references in Figure 1 turns to the lower side to directly select the virtual EMF as $d$-axis voltage reference, and the VSG control strategy becomes the same as in [8-14]. Then, according to the small-signal relationship shown in Figure 2, the small-signal models without the cascaded inner control loop can be obtained by applying the following simplification in (37) to the developed small-signal models $\mathbf{Y}_{V S G}$ in (32).

$$
G_{\psi 2 E}=\left[\begin{array}{l}
1 \\
0
\end{array}\right], G_{v 2 E}=\left[\begin{array}{ll}
0 & 0 \\
0 & 0
\end{array}\right], G_{i 2 E}=\left[\begin{array}{ll}
0 & 0 \\
0 & 0
\end{array}\right], G_{\theta 2 E}=\left[\begin{array}{l}
0 \\
0
\end{array}\right]
$$


Similar to (32), the simplified SISO impedance model of VSG controlled converters without cascaded control loop can be solved with (34) as:

$$
Z_{p}^{v s g, o p} \approx\left(s+j 2 \pi f_{1}\right) L_{f}-\frac{j}{2} \cdot \frac{\omega_{b a s e}}{J s^{2}+D_{p} s}-\frac{j}{2} \cdot \frac{K}{s}
$$

where $s=j 2 \pi\left(f_{p}-f_{1}\right)$.

For VSG controlled converter systems, the simplified SISO impedance model in (32) and the equivalent small-signal model (33) are shown in Figure 5, where the system and control parameters are kept the same as in Table 1. The connected grid is assumed inductive and the corresponding short circuit ratio (SCR) is set to 3. The impedance characteristics of VSG controlled converters without cascaded inner control loop are also plotted for comparison.

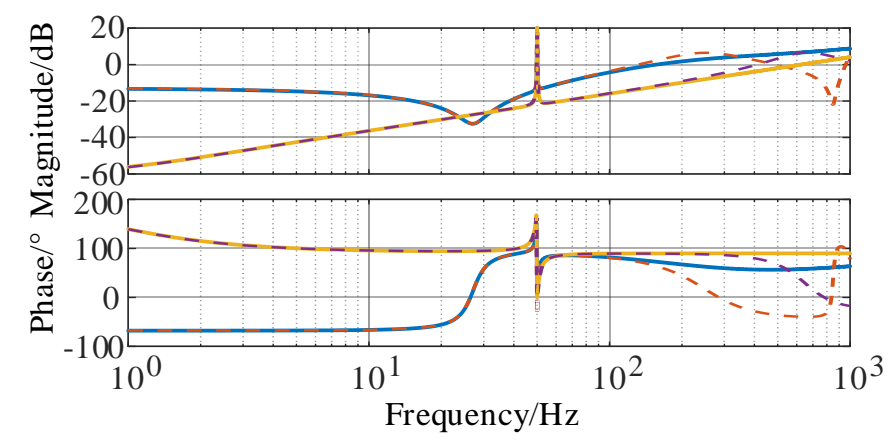

With cascaded control: — SISO impedance in (35) ----- equivalent small-signal models from (36) Without cascaded control:- SISO impedance in (38) ----- equivalent small-signal models from (36)(37)

Figure 5. Impedance characteristics of VSG controlled converters.

Figure 5 shows that, whether the cascaded inner control loop is adopted in the VSG strategy or not, the simplified SISO impedance models in (35) and (38) both accord with the corresponding equivalent small-signal models obtained from (36) in the low frequency range $(1-100 \mathrm{~Hz})$. Thus, the simplified SISO impedance model in (35) can be applied to identify the influence of the cascaded inner controller loop within the low frequency range in the following analysis.

\subsection{Impedance Characteristics of VSG Controlled Converters with Cascaded Inner Control Loop}

Figure 5 also shows that the application of cascaded inner control loop in the VSG strategy can significantly shapes the impedance characteristics of the VSG controlled converters. The impedance characteristics with cascaded inner control loop can be summarized as:

(1) The magnitude of VSG controlled impedance increases in the wide-band frequency range with the application of cascaded inner control loop.

(2) The impedance of VSG controlled converters can become capacitive below the fundamental frequency with the application of cascaded inner control loop, while the impedance without cascaded inner control loop is generally inductive.

\section{Influence Analysis of the Cascaded Inner Control Loop on Impedance Characteristics}

In this section, the capacitive impedance characteristics of the VSG controlled converters with cascaded inner control loop are concerned and the frequency range of interest is selected as the frequency below the fundamental frequency. The SISO impedance model in (32) is applied to identify the specific causes of capacitive impedance characteristics in VSG controlled converters. The influence of control parameters in the cascaded inner control loop is also quantified by differentiating the mathematical expression of the developed SISO impedance models in (32). 


\subsection{Causes of Capacitive Impedance Characteristics}

According to the simplified SISO impedance model in (32), the impedance model can be written as the sum of five parts, where the first three parts are related to the cascaded inner control loop with the virtual impedance and the last two parts are determined by the active and reactive power control. The real and imaginary expressions of (32) can be solved by substituting $s=j 2 \pi\left(f_{p}-f_{1}\right)$ into (32) to obtain equivalent resistance and reactance in p.u. with frequency $f_{p}$ variation as:

$$
\begin{aligned}
& R\left(f_{p}\right)=R_{v}+R_{h v}\left(f_{p}\right)+R_{h v h i}\left(f_{p}\right)+R_{\theta}\left(f_{p}\right)+R_{\psi}\left(f_{p}\right) \\
& X\left(f_{p}\right)=L_{v}+X_{h v}\left(f_{p}\right)+X_{h v h i}\left(f_{p}\right)+X_{\theta}\left(f_{p}\right)+X_{\psi}\left(f_{p}\right)
\end{aligned}
$$

where $R_{v}$ and $L_{v}$ are constant virtual resistance and inductance. Expressions of $R_{h v}\left(f_{p}\right), R_{h v h i}\left(f_{p}\right), X_{h v}\left(f_{p}\right)$, and $X_{h v h i}\left(f_{p}\right)$, which are related to the cascaded inner control loop, are listed below with $\omega=2 \pi\left(f_{p}-f_{1}\right)$.

$$
\begin{aligned}
& R_{h v}\left(f_{p}\right)=\frac{\omega^{2} k_{v p}^{2}}{\omega^{2} k_{v p}^{2}+k_{v i}^{2}}, R_{h v h i}\left(f_{p}\right)=\frac{-\omega^{4} L_{f}\left(k_{v i} k_{i p}+k_{v p} k_{i i}\right)}{\left(k_{v i} k_{i i}-\omega^{2} k_{v p} k_{i p}\right)^{2}+\omega^{2}\left(k_{v i} k_{i p}+k_{v p} k_{i i}\right)^{2}} \\
& X_{h v}\left(f_{p}\right)=\frac{\omega k_{v i}^{2}}{\omega^{2} k_{v p}^{2}+k_{v i}^{2}}, X_{h v h i}\left(f_{p}\right)=\frac{-\omega^{3} L_{f}\left(k_{v i} k_{i i}-\omega^{2} k_{v p} k_{i p}\right)}{\left(k_{v i} k_{i i}-\omega^{2} k_{v p} k_{i p}\right)^{2}+\omega^{2}\left(k_{v i} k_{i p}+k_{v p} k_{i i}\right)^{2}}
\end{aligned}
$$

According to (36)-(38), results of equivalent resistance and reactance are plotted in Figure 6, where parameters are the same as in Figure 5.

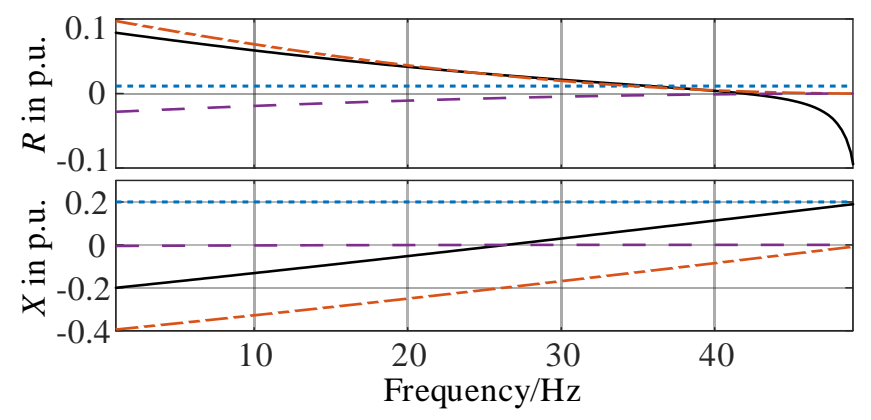

$$
-R\left(f_{p}\right) \text { and } X\left(f_{p}\right) \cdots R_{v} \text { and } L_{v}=\cdots R_{h v}\left(f_{p}\right) \text { and } X_{h v}\left(f_{p}\right)-\cdots R_{h v h i}\left(f_{p}\right) \text { and } X_{h v h i}\left(f_{p}\right)
$$

Figure 6. Equivalent resistance and reactance in p.u. of VSG controlled converters with cascaded inner control loop.

By comparing the equivalent resistance and reactance in (36) with the results obtained from (37)-(38), conclusions about the causes of capacitive characteristics can be reached from the results in Figure 6:

(1) Since $\omega=2 \pi\left(f_{p}-f_{1}\right)<0$ when $f_{p}<f_{1}$, the reactance obtained from $X_{h v}\left(f_{p}\right)$ in (38) stays negative below the fundamental frequency, which means that the capacitive impedance characteristics are mainly caused by the effects of voltage controller in the cascaded control loop.

(2) The resistance obtained from $R_{h v h i}\left(f_{p}\right)$ in (37) are negative below the fundamental frequency, which means the control effects of the cascaded voltage-current controller tend to cause negative resistance in this frequency range.

(3) The resistance and reactance obtained from the $R_{v}$ and $L_{v}$ are both positive, which means the virtual impedance control can both reduce negative resistive and capacitive characteristics by choosing positive virtual resistance and virtual inductance. 


\subsection{Influence of Control Parameters in Cascaded Inner Control Loop}

In the following, the simplified SISO impedance model in (32) is differentiated with respect to each related control parameter $x$, and the real and imaginary parts of this differential expression are calculated to refer to the influence on the equivalent resistance and reactance of the impedance characteristics as:

$$
\left(\frac{d R}{d x}\right)_{p . u .}=\operatorname{Re}\left\{x \cdot \frac{d Z_{p}^{v s g}}{d x}\right\},\left(\frac{d X}{d x}\right)_{p . u .}=\operatorname{Im}\left\{x \cdot \frac{d Z_{p}^{v s g}}{d x}\right\}
$$

Based on the differential expression in (39), the absolute value of (39) can represent the influence ability of this parameter in p.u., and the sign of the real and imaginary part reflects the influence direction of this parameter.

For example, if $(d R / d x)_{p . u .}<0$ and $|d R / d x|_{p . u}$. is not close to zero in a certain frequency range, it represents that decreasing control parameter $x$ will increase the equivalent resistance (equally to reduce the negative resistive characteristics) in this frequency range.

Similarly, if $(d X / d x)_{p . u .}>0$ and $|d X / d x|_{p . u .}$ is larger than zero in a certain frequency range, increasing control parameter $x$ will increase the equivalent inductance (equally to reduce the capacitive impedance characteristics) in this frequency range.

The gain and integrator coefficients of the cascaded voltage controller and current controller are calculated with the differential analysis in (39), respectively, and the differential results below the fundamental frequency are given in Figure 7, from which it can be observed that:

(1) The negative resistive characteristics below the fundamental frequency can be reduced by increasing $k_{i p}$ and $k_{v p}$, or by decreasing $k_{v i}$, and the influencing ability of $k_{i i}$ is as not significant as the other control parameters in cascaded control loop.

(2) The capacitive impedance characteristics below the fundamental frequency can be reduced by increasing $k_{v p}$ and $k_{v i}$, and the influence ability of current controller is not as significant as the voltage controller.
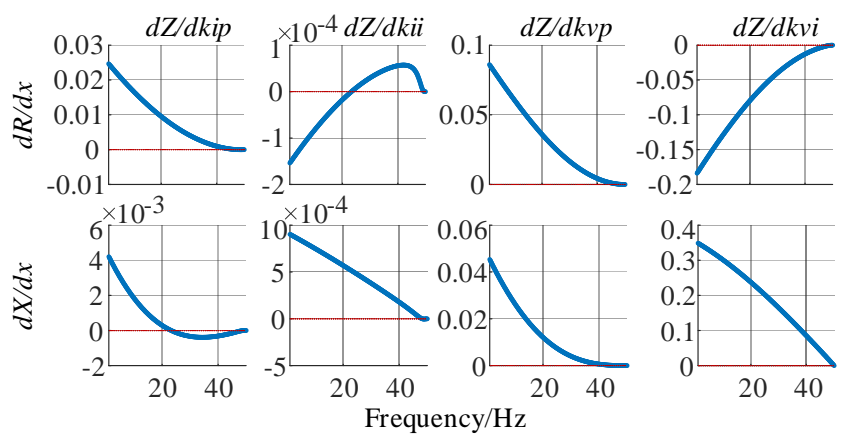

Figure 7. Influence of cascaded voltage-current control parameters on the impedance characteristics of VSG controlled converters.

\section{Impedance-Based System Stability Analysis of VSG Controlled Converters with Weak Grid}

In this section, impedance-based stability analysis is carried out to analyze the possible system instability of VSG controlled converters with the different kinds of weak grid. The influence of the cascaded inner control loop on the system stability are also provided by changing the control parameters. CHIL-based experiments were also carried out to validate the instability cases.

\subsection{Possible System Instability with Different Weak Grid}

The system stability of converters and the connected grid can be analyzed with impedance models. According to the impedance characteristics of VSG controlled converters, the following two conditions with different kinds of weak grid may lead to possible system instability: 
(1) When connecting with the inductive weak grid, the capacitive impedances characteristics that shaped by the cascaded inner control loop may be incompatible with the inductive weak grid, especially when there is negative resistance in the capacitive frequency range.

(2) When connecting with the series-compensated weak grid, the inductive impedance characteristics of converters may be unmatched with the capacitive grid, while the capacitive impedances characteristics shaped by the cascaded inner control loop can match the capacitive grid better.

In the following, two cases are provided to verify above two possibilities, and the influence of cascaded inner control loop on these possible instabilities are verified by changing the control parameters. CHIL-based experiments were also carried out to validate these cases.

\subsection{System Instability with Inductive Weak Grid-A Case Study}

In this subsection, a case to verify the possible instability of VSG controlled converters with inductive weak grid is provided. In this case, the parameter $k_{i p}$ is decreased from 1.0 to 0.2 p.u., while other parameters are unchanged as in Table 1 and the SCR of the inductive weak grid is kept as 3.

The impedance characteristics of VSG controlled converters with the inductive weak grid and the corresponding Nyquist plot are given in Figure 8. Figure 8a shows that, when adopting the VSG control strategy with cascaded inner control loop, the phase below the fundamental frequency decreases after decreasing the $k_{i p}$, which accords with the analysis in Section 4.2 that the negative resistance in this frequency range can be reduced by increasing $k_{i p}$. In contrast, the impedance of VSG controlled converters without adopting cascaded control loop is generally inductive in the wide frequency range, and accords with the inductive grid impedance, as shown in Figure 8a.

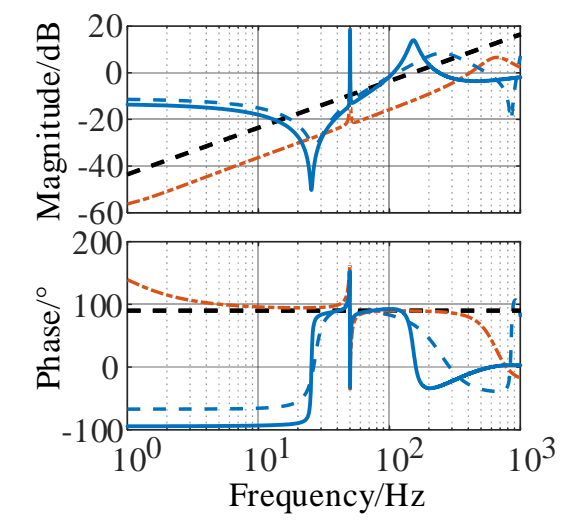

---- VSG with cascaded control, $k_{i p}=1.0$ p.u.

— VSG with cascaded control, $k_{i p}=0.2$ p.u.

VSG without cascaded control

--- $\quad$ grid impedance $(\mathrm{SCR}=3)$

(a)

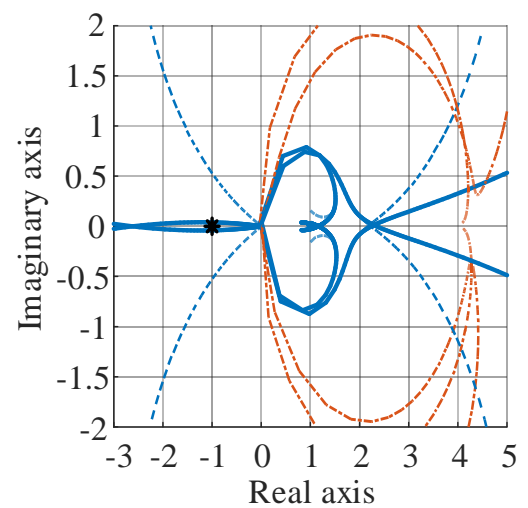

---- VSG with cascaded control, $k_{i p}=1.0$ p.u. VSG with cascaded control, $k_{i p}=0.2$ p.u. VSG without cascaded control

(b)

Figure 8. Stability of VSG controlled converters with the inductive weak grid: (a) impedance characteristics; and (b) Nyquist plot.

In Figure 8b, the Eigen loci of VSG control strategy without cascaded inner control loop are away from $(-1, j 0)$ point, while the Eigen loci of VSG control strategy with cascaded inner control loop circle the $(-1, j 0)$ point at the frequency about $15 \mathrm{~Hz}$ after decreasing the $k_{i p}$ from 1.0 to 0.2 p.u., which predicts that the system becomes unstable when $k_{i p}$ decreases to 0.2 p.u. in this case.

Time-domain simulations based on Simulink and CHIL-based experiments were both carried out to verify above system stability analysis. In simulations and experiments, parameters of VSG control strategy and the SCR of the inductive grid were kept the same as the former impedance-based stability analysis, and the control parameter $k_{i p}$ changed from 1.0 to 0.2 p.u. to cause this instable case after the 
VSG controlled converter with the cascaded inner control loop reached a stable operating state and generated about $1.375 \mathrm{MW}$ active power to the connected weak grid.

In simulations, parameter $k_{i p}$ changed from 1.0 to 0.2 p.u. at $10.5 \mathrm{~s}$. The waveforms of active power and reactive power are plotted in Figure 9, where resonances at $36 \mathrm{~Hz}$ (fundamental $50 \mathrm{~Hz}$ minus $14 \mathrm{~Hz}$ ) can be observed in the active and reactive power, and the amplitude of resonances increases with time.

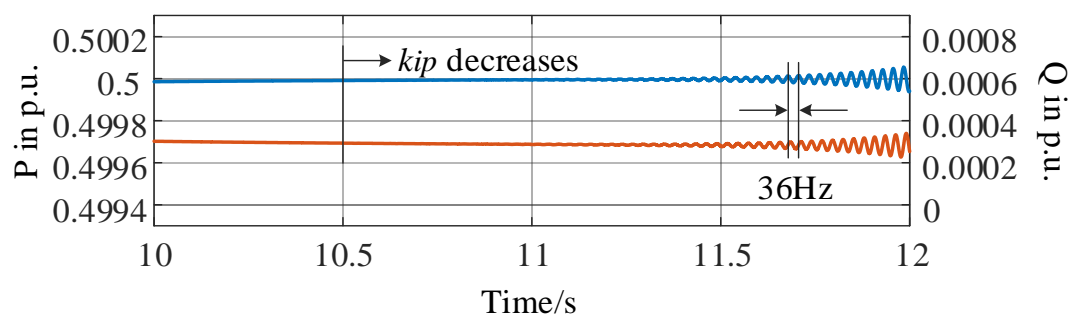

Figure 9. Simulation waveforms of active power and reactive power in p.u. in the VSG controlled converter when $k_{i p}$ decreases (blue lines, active power; orange lines, reactive power).

In CHIL-based experiments, the control parameter $k_{i p}$ changed from 1.0 to 0.2 p.u. and then changed back to 1.0 p.u. after $0.5 \mathrm{~s}$. The system current in phase-a/b/c and the active and reactive power generated from the VSG controlled converter to the inductive weak grid are plotted in Figure 10.

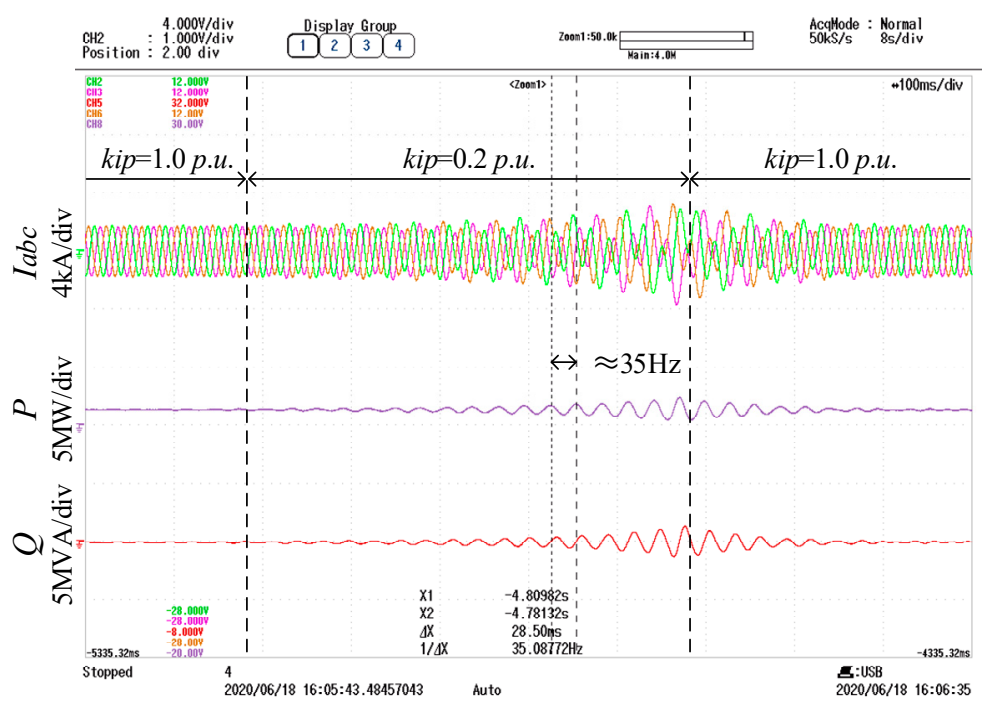

Figure 10. Waveforms of system current and active and reactive power when $k_{i p}$ decreases.

Figure 10 shows that, before decreasing the parameter $k_{i p}$ in the cascaded control loop, the VSG controlled converter operates stably with the connected weak grid. When the parameter $k_{i p}$ decreases to 0.2 p.u., resonances arise in waveforms of system current, active power, and reactive power, and resonances are suppressed when the parameter $k_{i p}$ increases back to $1.0 \mathrm{p} . \mathrm{u}$. Besides, resonances in the active and reactive power are at around $35 \mathrm{~Hz}$ (fundamental $50 \mathrm{~Hz}$ minus $15 \mathrm{~Hz}$ ), which accords with above impedance-based stability analysis that the impedance characteristics of the VSG controlled converter and the inductive weak grid are mismatched with a small parameter $k_{i p}$ since the corresponding Eigen loci circle the $(-1, j 0)$ point at the frequency about $15 \mathrm{~Hz}$, as shown in Figure 8b.

It should be noted that the control of the converter was simulated in the Simulink platform, while controllers were implemented on a TMS320F28335 DSP+FPGA control board and with the sampling and switching frequency set as $5 \mathrm{kHz}$ in CHIL-based experiments. Thus, the control effects of the hardware controller applied in the experiments and the simulated controller in simulations 
were slightly different due to the sampling error, control delay and other factors, which then led to different impedance characteristics of VSG controlled converters in experiments and simulations to some extent. Therefore, the resonant frequencies in experiments and simulations are slightly different in Figures 9 and 10. However, even though there are some slight differences from simulation results, the experimental results in this manuscript are still valid and accord with the theoretical impedance-based stability analysis.

It should also be noted that decreasing $k_{i p}$ to 0.2 p.u. is an example to explain that the operation of VSG controlled converters may be unstable with the inductive weak grid due to the capacitive impedance characteristics shaped by the cascaded inner control loop. According to the impedance-based analysis in Section 4.2 , the instability can also be avoided by increasing $k_{v p}, k_{i p}$, and $R_{v}$ to increase the equivalent resistance in the capacitive frequency range.

\subsection{System Instability with Series-Compensated Weak Grid-A Case Study}

In this subsection, a case to verify the possible instability of VSG controlled converters with series-compensated weak grid is provided. Parameters of VSG control strategy are kept the same as in Table 1, and the compensated level of the series-compensated grid is set as $10 \%$. Impedance characteristics and Nyquist plot of the grid-converter system are given in Figure 11.

In Figure 11a, the impedance characteristics of the VSG controlled converters with cascaded control loop and the compensated grid both can behave capacitive below the fundamental frequency, while the magnitude curves of VSG strategy without cascaded inner control loop and the series-compensated grid intersect at the frequency about $13 \mathrm{~Hz}$, and the phase difference between the grid and the converter is around 180 degrees.

As shown in Figure 11b, the Eigen loci of VSG strategy without cascaded inner control loop cross the $(-1, j 0)$ point at the frequency about $13 \mathrm{~Hz}$, while the Eigen loci with cascaded inner control loop keep away from the $(-1, j 0)$ point. This system stability analysis predicts that, when connecting with this series-compensated grid, the application of cascaded inner control loop can avoid causing resonances in the interconnected system.

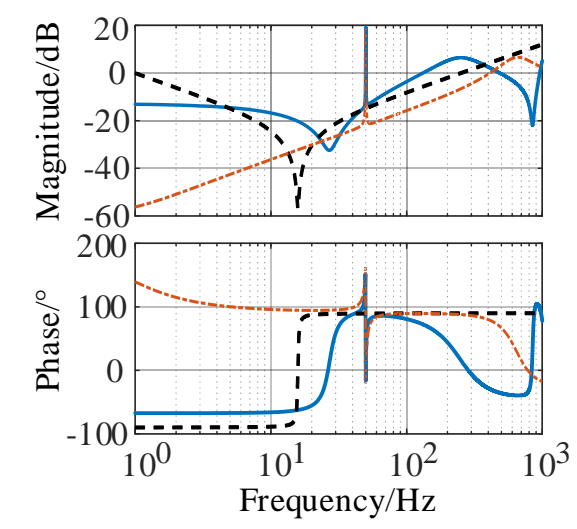

- VSG with cascaded control

-.--.-. VSG without cascaded control

--- grid impedance (compensated level 10\%)

(a)

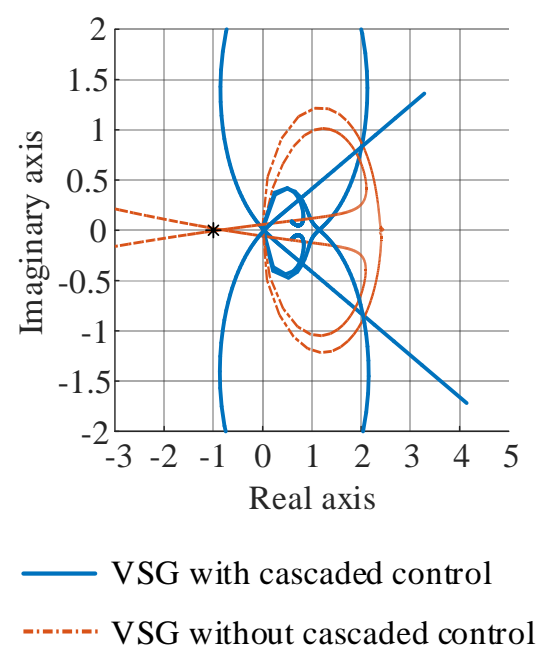

(b)

Figure 11. Stability of VSG controlled converters with series-compensated grid: (a) impedance characteristics; and (b) Nyquist plot.

The above analysis can be verified by both time-domain simulations based on Simulink and CHIL-based experiments. In simulations and experiments, parameters of VSG control strategy and the compensated level of the series-compensated grid were kept the same as the former impedance-based 
stability analysis shown in Figure 11, and active power reference of the VSG controlled converter was set as $1.375 \mathrm{MW}$. When the grid-converter system reached the stable operating state, the cascaded inner control loop was disabled and then re-enabled later to compare the different system stability of VSG controllers with and without cascaded inner control loop.

In simulations, the cascaded control loop was disabled at $6 \mathrm{~s}$ and re-enabled at $7 \mathrm{~s}$. The waveforms of PCC current in phase- $a$, the active power, and reactive power are plotted in Figure 12. FFT analysis was applied to the current waveform from 6 to $7 \mathrm{~s}$, and the FFT results are plotted in Figure 13. Figure 12 shows that, when the cascaded inner control loop was disabled at $6 \mathrm{~s}$, resonances were caused, and, when the cascaded inner control loop was re-enabled at $7 \mathrm{~s}$, resonances were suppressed. In Figure 13, the most significant resonant components of the system current in phase- $a$ from 6 to $7 \mathrm{~s}$ are at frequencies 13 and $14 \mathrm{~Hz}$.

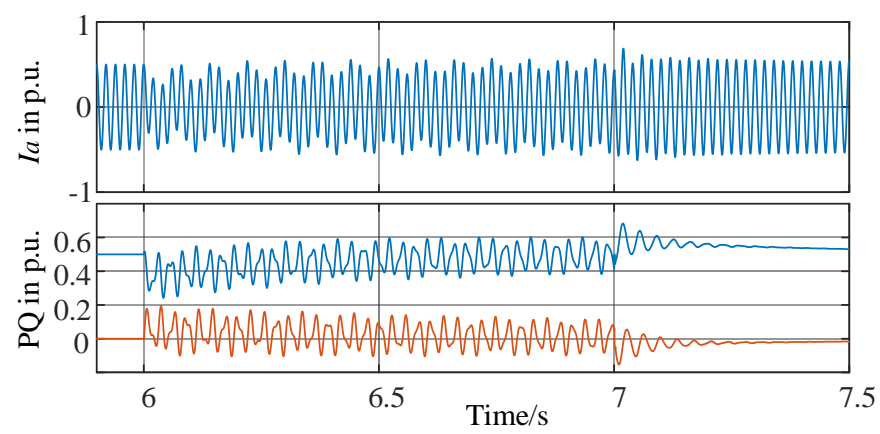

Figure 12. Simulation waveforms when disable/enable the cascaded control loop in the VSG controlled converter with the series-compensated grid: (top) system current in phase-a; and (bottom) the blue line is active power and the orange line is reactive power.

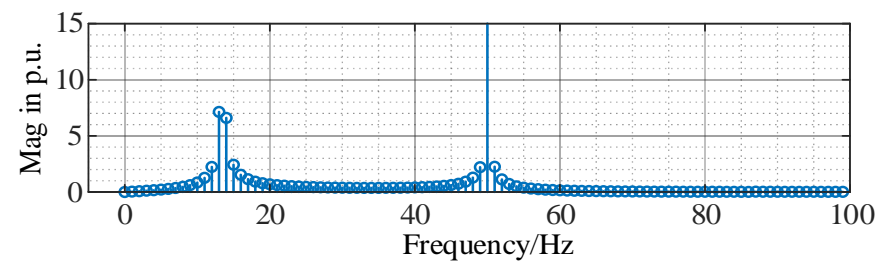

Figure 13. FFT analysis of resonant current in grid-converter interconnected system.

In CHIL-based experiments, the system current in phase-a/b/c, active power and reactive power generated from the VSG controlled converter to the compensated weak grid are plotted in Figure 14. Figure 14 shows that, before disabling the cascaded inner control loop in the VSG strategy, the VSG controlled converter operated stably with generating about 1.375 MW active power to the compensated weak grid. When the switch of modulation references turned to the lower side, as shown in Figure 1, to directly select the virtual EMF as d-axis modulation reference, the cascaded inner control loop was disabled in this VSG strategy. In Figure 14, when the cascaded inner control loop is disabled, resonances are caused in the waveforms of system currents, active power, and reactive power, and the resonances gradually reduce when the cascaded control loop is re-enabled. Besides, the resonances in the active and reactive power are about $37 \mathrm{~Hz}$ (fundamental $50 \mathrm{~Hz}$ minus $13 \mathrm{~Hz}$ ), which accords with the above stability analysis in Figure 11 that the impedance characteristics of the VSG controlled converters without cascaded control loop are mismatched with the impedance characteristics of compensated weak grid at $13 \mathrm{~Hz}$.

Therefore, different from the possibly poor system stability with the inductive weak grid, the capacitive impedance characteristics of the VSG controlled converters with the cascaded control loop match the series-compensated weak grid better. This case study can also be seen as an example to explain the possible advantage of adopting the cascaded inner control loop in VSG strategy. 


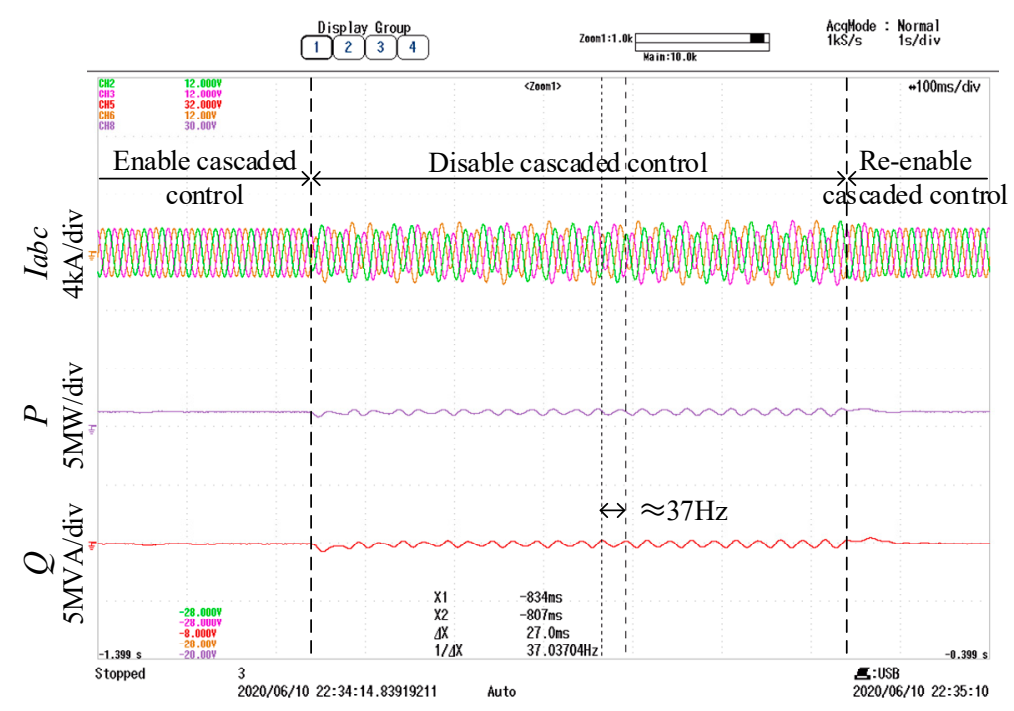

Figure 14. Waveforms of disable and re-enable the cascaded inner control loop in VSG control strategy with series-compensated grid.

\section{Conclusions}

In this paper, a SISO impedance model with simple mathematical expression is obtained from the small-signal modeling of the VSG controlled converters with cascaded inner control loop. Based on the simplified SISO impedance model, the impedance shaping effects of cascaded inner control loop are identified, and the influence of control parameters on system stability are quantified by differential analysis. Possible system instabilities of VSG controlled converters with different kinds of weak grid are revealed and validated with CHIL-based experimental cases.

The main conclusions are summarized as follows:

(1) The impedance characteristics of VSG controlled converters with cascaded inner control loop can become capacitive below the fundamental frequency, which is mainly caused by the voltage controller in the cascaded control loop. The cascaded voltage and current controller with the virtual impedance can also shape the capacitive and negative resistive characteristics in this frequency range.

(2) The capacitive impedance characteristics can benefit the compatibility of converters operated with the series-compensated weak grid but may deteriorate the system stability with the inductive weak grid. Possible system instabilities can be avoided by increasing control parameter $k_{v p}, k_{i p}$, and virtual resistance $R_{v}$ to reduce negative resistance in the capacitive frequency range.

Author Contributions: Conceptualization, Y.X. and H.N.; data curation, Y.X.; formal analysis, Y.X.; funding acquisition, H.N. and D.S.; investigation, Y.X.; methodology, Y.X.; project administration, H.N.; resources, H.N. and D.S.; software, Y.X.; supervision, H.N.; validation, Y.X.; visualization, Y.X. and Y.W.; writing-original draft preparation, Y.X.; and writing-review and editing, H.N. and Y.W. All authors have read and agreed to the published version of the manuscript.

Funding: This research was supported in part by National Natural Science Foundation of China under Grant 51977194 and in part by Zhejiang Provincial Natural Science Foundation of China under grant LZ18E070001.

Conflicts of Interest: The authors declare no conflict of interest. 


\section{Nomenclature}

$\mathrm{V}, \mathrm{I}, \mathrm{E} \quad$ voltage, current, and modulation signals

$\mathrm{P}, \mathrm{Q},|\mathrm{V}| \quad$ active power, reactive power, and voltage magnitude

abc, dq, dc subscripts indicating variables are in a/b/c-phase, on d/q-axis, or on dc side

ref subscript indicating variables are reference variables

$L_{f}, C_{f}, R_{f} \quad$ filter inductor, filter capacitor, and filter resistor of the converter

$\mathrm{V}_{\mathrm{g}, \mathrm{Zg}} \quad$ grid voltage and grid impedance

$\psi_{\mathrm{f}} \quad$ virtual back electrical magnetic field

$\theta \quad$ virtual rotor angular position

$\omega_{\text {pu }} \quad$ per unit angular speed

$\omega_{\mathrm{g}, \mathrm{pu}} \quad$ per unit angular frequency of the connected grid

$\mathrm{J}$ inertial constant

$\mathrm{D}_{\mathrm{p}} \quad$ damping coefficient of active power

$\mathrm{D}_{\mathrm{q}} \quad$ voltage droop coefficient

$\mathrm{K}$ integral coefficient of reactive power controller

$\mathrm{L}_{\mathrm{V}}, \mathrm{R}_{\mathrm{V}} \quad$ virtual inductor and virtual resistor

$\mathrm{K}_{\mathrm{FFi}}, \mathrm{K}_{\mathrm{FFv}} \quad$ feedforward gain of current and voltage

$\mathrm{k}_{\mathrm{vp}}, \mathrm{k}_{\mathrm{vi}} \quad$ proportional and integral coefficient of cascaded voltage controller

$\mathrm{k}_{\mathrm{ip}}, \mathrm{k}_{\mathrm{ii}} \quad$ proportional and integral coefficient of cascaded current controller

$\mathrm{H}_{\mathrm{v}}(\mathrm{s}), \mathrm{H}_{\mathrm{i}}(\mathrm{s}) \quad$ transfer functions of the cascaded voltage and current controller

$\hat{\mathbf{X}} \quad$ bold variables with symbol ^ above indicating variables are perturbed frequency components

$1, p, p 2 \quad$ subscripts indicating components at fundamental frequency, frequency $f_{p}$, and frequency $f_{p}-2 f_{1}$

$\mathrm{G}_{\mathrm{x} 2 \mathrm{y}} \quad$ small-signal blocks to describe the small-signal relationships between variables $\mathrm{x}$ and $\mathrm{y}$

$\mathrm{T}_{\mathrm{m}, \mathrm{n}} \quad$ abbreviations of transfer matrices between variables $\mathrm{m}$ and $\mathrm{n}$

$\mathrm{Y}_{\mathrm{VSG}} \quad$ small-signal models of VSG controlled converters in $2 \times 2$ order admittance matrix

$\mathrm{Y}_{\mathrm{cf}} \quad 2 \times 2$ order admittance matrix model of the filter resistor $\mathrm{R}_{\mathrm{f}}$ and filter capacitor $\mathrm{C}_{\mathrm{f}}$

$Z_{p}^{v s g} / Z_{p}^{v s g, o p} \quad S I S O$ impedance model for VSG controlled converters with/without the cascaded control loop

$Y_{p}^{e q l} / Z_{p}^{e q l} \quad$ equivalent admittance/impedance of the small-signal models

VSG virtual synchronous generator

SISO single-in-single-out

CHIL control-hardware-in-loop

SG synchronous generator

REGS renewable energy generation system

PLL phase-locked loop

EMF virtual back electrical magnetic field

PCC point of common coupling

\section{Appendix A}

Based on the small-signal modeling in Section 2, the small-signal models of the VSG controlled converter can be described by a $2 \times 2$ order admittance matrix $\mathbf{Y}_{V S G}$ in (32), whose detailed expressions can be obtained by substituting the detailed expressions of each blocks $G_{x 2 y}$ as given in Section 2.3.

Then, with the simplification in Steps 1-3 in Section 3.1, the impedance of VSG controlled converters can be simplified as:

$$
Z_{s i m p 1}=\frac{1-\mathbf{M}_{i}(1,1)}{\mathbf{M}_{v}(1,1)}=\frac{Z_{v} H_{v} H_{i}+H_{i}+\left(s-j \omega_{1}\right) L_{f}+\frac{j}{2} F_{\psi, i}(s) \cdot G_{q 2 \psi}+\frac{j}{2} F_{\theta, i}(s) \cdot G_{p 2 \theta}}{\left(H_{v}-j \omega_{1} C_{f}\right) H_{i}-\frac{j}{2} F_{\psi, v}(s) \cdot G_{q 2 \psi}+\frac{j}{2} F_{\theta, v}(s) \cdot G_{p 2 \theta}}
$$

where $Z_{v}=R_{v}+j L_{v}$ and expressions of transfer functions $F_{\psi, i}, F_{\psi, v}, F_{\theta, I}$, and $F_{\theta, v}$ are: 


$$
\begin{aligned}
& F_{\psi, i}(s) \approx H_{v}(s) H_{i}(s), F_{\psi, v}(s) \approx H_{v}(s) H_{i}(s)\left(I_{1} e^{-j \varphi i 1+j \theta 0}-j D_{q} V_{1}\right) \\
& F_{\theta, i}(s) \approx\left(H_{v}(s)-j \omega_{1} C_{f}\right) H_{i}(s)+\left(H_{i}(s)-j \omega_{1} L_{f}\right)\left(I_{g 1} e^{-j \varphi v 1+j \varphi i g 1}\right)+Z_{v} H_{v}(s) H_{i}(s)\left(I_{1} e^{-j \varphi v 1+j \varphi i 1}\right) \\
& F_{\theta, v}(s) \approx\left(H_{v}(s)-j \omega_{1} C_{f}\right) H_{i}(s)\left(I_{1} e^{-j \varphi i 1+j \varphi v 1}\right)+\left(H_{i}(s)-j \omega_{1} L_{f}\right)\left(I_{1} I_{g 1}\right)+Z_{v} H_{v}(s) H_{i}(s)\left(I_{1} I_{1}\right)
\end{aligned}
$$

For VSG controlled converters, the control bandwidths of the active and reactive power controllers $(1-2 \mathrm{~Hz})$ are much smaller than the cascaded voltage controller and the cascaded current controller (50-200 Hz). Then, according to (A2), the denominator of (A2) is dominated by the multiplication of cascaded transfer function $H_{v}(s){ }^{*} H_{i}(s)$ and the expressions of power controllers $G_{p 2 \theta}$ and $G_{q 2 \psi}$ in the denominator can be simplified to zero, which means (A2) is further simplified as follows:

$$
Z_{\text {simp } 2} \approx \frac{Z_{v} H_{v} H_{i}+H_{i}+\left(s-j \omega_{1}\right) L_{f}+\frac{j}{2} F_{\psi, i}(s) G_{q 2 \psi}+\frac{j}{2} F_{\theta, i}(s) G_{p 2 \theta}}{\left(H_{v}-j \omega_{1} C_{f}\right) H_{i}}
$$

Besides, within the control bandwidth of the cascaded voltage controller ( $54 \mathrm{~Hz}$ in this manuscript), the value of $H_{v}(s) * H_{i}(s)$ is larger than $H_{v}(s)$. Then, within the frequency range $54 \mathrm{~Hz}$ below the fundamental frequency to $54 \mathrm{~Hz}$ above the fundamental frequency (1-100 Hz for convenience), the expression of transfer function $F_{\theta, i}(s)$ in (A2) can be further simplified as:

$$
F_{\theta, i}(s) \approx\left(H_{v}(s)-j \omega_{1} C_{f}\right) H_{i}(s)+Z_{v} H_{v}(s) H_{i}(s)\left(I_{1} e^{j \varphi i 1}\right)
$$

Furthermore, the decoupling gain of voltage controller in (A3) and (A4) is ignored, and substituting the above-simplified $F_{\theta, i}(s)$ to (A3), (A3) is then simplified as:

$$
\begin{aligned}
Z_{\text {simp } 3} & \approx Z_{v}+\frac{1}{H_{v}(s)}+\frac{s L_{f}}{H_{v}(s) H_{i}(s)}+\frac{j}{2} G_{q 2 \psi}+\frac{j}{2}\left(1+Z_{v} I_{1}\right) G_{p 2 \theta} \\
& \approx Z_{v}+\frac{1}{H_{v}(s)}+\frac{s L_{f}}{H_{v}(s) H_{i}(s)}+\frac{j}{2} G_{q 2 \psi}+\frac{j}{2} G_{p 2 \theta}, \text { since } Z_{v} I_{1} \ll 1 \text { in this case }
\end{aligned}
$$

Finally, substituting the detailed expressions of $G_{p 2 \theta}$ and $G_{q 2 \psi}$ to (A5), the simplified SISO impedance model of VSG controlled converters can be obtained as (35) in Section 3.1.

\section{References}

1. Beck, H.-P.; Hesse, R. Virtual synchronous machine. In Proceedings of the 9th International Conference on Electrical Power Quality and Utilization, Barcelona, Spain, 9-11 October 2007; pp. 1-6.

2. Subsynchronous Resonance Working Group of the System Dynamic Performance Subcommittee. Reader's guide to subsynchronous resonance. IEEE Trans. Power Syst. 1992, 7, 150-157. [CrossRef]

3. Liu, H.; Xie, X.; He, J.; Xu, T.; Yu, Z.; Wang, C.; Zhang, C. Subsynchronous interaction between direct-drive PMSG based wind farms and weak ac grid. IEEE Trans. Power Syst. 2017, 32, 4708-4720. [CrossRef]

4. Shu, D.; Xie, X.; Rao, H.; Gao, X.; Jiang, Q.; Huang, Y. Sub- and super- synchronous interactions between STATCOMs and weak AC/DC transmissions with series compensations. IEEE Trans. Power Electron. 2018, 33, 7424-7437. [CrossRef]

5. Chen, X.; Zhang, Y.; Wang, S.; Chen, J.; Gong, C. Impedance-phased dynamic control method for grid-connected inverters in a weak grid. IEEE Trans. Power Electron. 2017, 32, 274-283. [CrossRef]

6. Zhang, B.; Du, X.; Zhao, J.; Zhou, J.; Zou, X. Impedance modeling and stability analysis of a three-phase three-level NPC inverter connected to the grid. CSEE J. Power Energy Syst. 2020, 6, 270-278.

7. Sun, J. Impedance-based stability criterion for grid-connected inverters. IEEE Trans. Power Electron. 2011, 26, 3075-3078. [CrossRef]

8. Wu, H.; Ruan, X.; Yang, D.; Chen, X.; Zhao, W.; Lv, Z.; Zhong, Q. Small-signal modeling and parameters design for virtual synchronous generators. IEEE Trans. Ind. Electron. 2016, 63, 4292-4303. [CrossRef]

9. Mo, O.; D'Acro, S.; Suul, J.A. Evaluation of virtual synchronous machines with dynamic or quasi-stationary machine models. IEEE Trans. Ind. Electron. 2017, 64, 5952-5962. [CrossRef] 
10. Du, W.; Fu, Q.; Wang, H.F. Power system small-signal angular stability affected by virtual synchronous generators. IEEE Trans. Power Syst. 2019, 34, 3209-3219. [CrossRef]

11. Li, G.; Chen, Y.; Luo, A.; He, Z.; Wang, H.; Zhu, Z.; Wu, W.; Zhou, L. Analysis and mitigation of subsynchronous resonance in series-compensated grid-connected system controlled by a virtual synchronous generator. IEEE Trans. Power Electron. 2020, 35, 11096-11107. [CrossRef]

12. Yu, Y.; Zhang, M.; Antoine, M.; Li, H. Analysis of synchronous frequency resonance in VSG based on the sequence impedance models. In Proceedings of the 22nd International Conference on Electrical Machines and Systems, Harbin, China, 11-14 August 2019; pp. 1-6.

13. Zhang, X.; Chen, J.; Chen, X. Impedance modeling and stability analysis of weak-grid interfaced single-phase VSG. In Proceedings of the 22nd International Conference on Electrical Machines and Systems, Harbin, China, 11-14 August 2019; pp. 1-6.

14. Wu, W.; Zhou, L.; Chen, Y.; Luo, A.; Dong, Y. Sequence-impedance-based stability comparison between VSGs and traditional grid-connected inverters. IEEE Trans. Power Electron. 2019, 34, 46-52. [CrossRef]

15. Zhong, Q.; Weiss, G. Synchronverters: Inverters that mimic synchronous generators. IEEE Trans. Ind. Electron. 2011, 58, 1259-1267. [CrossRef]

16. Wang, X.; Chen, L.; Sun, D.; Zhang, L.; Nian, H. A modified self-synchronized synchronverter in unbalanced power grids with balanced currents and restrained power ripples. Energies 2019, 12, 923. [CrossRef]

17. D'Arco, S.; Suul, J.A. Virtual synchronous machines-Classification of implementations and analysis of equivalence to droop controllers for microgrids. In Proceedings of the 2013 IEEE Grenoble Conference, Grenoble, France, 16-20 June 2013; pp. 1-7.

18. D'Arco, S.; Suul, J.A.; Fosso, O.B. A virtual synchronous machine implementation for distributed control of power converters in smart grids. Electr. Power Syst. Res. 2015, 122, 180-197. [CrossRef]

19. Hou, X.; Sun, Y.; Zhang, X.; Lu, J.; Wang, P.; Guerrero, J.M. Improvement of frequency regulation in VSG-based ac microgrid via adaptive virtual inertia. IEEE Trans. Power Electron. 2020, 35, 1589-1602. [CrossRef]

20. Chen, J.; Liu, M.; Milano, F.; O’Donnell, T. Placement of virtual synchronous generator controlled electric storage combined with renewable generation. In Proceedings of the IEEE Milan PowerTech, Milan, Italy, 23-27 June 2019; pp. 1-6.

21. Unamuno, E.; Rygg, A.; Amin, M.; Barrena, J.A. Impedance-based stability evaluation of virtual synchronous machine implementations in converter controllers. In Proceedings of the International Power Electronics Conference, Niigata, Japan, 20-24 May 2018; pp. 759-766.

22. Li, C.; Liang, J.; Cipcigan, L.M.; Ming, W.; Colas, F.; Guillaud, X. DQ impedance stability analysis for the power-controlled grid-connected inverter. IEEE Trans. Energy Convers. 2020, 1. [CrossRef]

23. Xu, Y.; Nian, H.; Xu, G.; Qiu, J. Cross-coupling over frequency and sequence in impedance modelling of grid-connected inverter. J. Eng. 2017, 2017, 990-995. [CrossRef]

24. Xu, Y.; Nian, H.; Wang, T.; Chen, L.; Zheng, T. Frequency coupling characteristic modeling and stability analysis of doubly fed induction generator. IEEE Trans. Energy Convers. 2018, 33, 1475-1486. [CrossRef]

25. Nian, H.; Xu, Y.; Chen, L.; Zhu, M. Modeling and analysis of dc-link dynamics in DFIG system with an indicator function. IEEE Access 2019, 7, 125401-125412. [CrossRef]

26. Zou, X.; Du, X.; Tai, H. Two-variable admittance model for D-PMSG-based wind turbine and stability criterion based on magnitude and phase contour plot. IEEE Trans. Power Electron. 2020, 35, 1484-1498. [CrossRef]

27. Liu, W.; Xie, X.; Zhang, X.; Li, X. Frequency-coupling admittance modeling of converter-based wind turbine generators and the control-hardware-in-the-loop validation. IEEE Trans. Energy Convers. 2020, 35, 425-433. [CrossRef]

28. Li, G.; Sun, J. Control hardware-in-the-loop simulation for turbine impedance modeling and verification. In Proceedings of the 16th Wind Integration Workshop, Berlin, Germany, 16-18 October 2017; pp. 1-6.

(C) 2020 by the authors. Licensee MDPI, Basel, Switzerland. This article is an open access article distributed under the terms and conditions of the Creative Commons Attribution (CC BY) license (http://creativecommons.org/licenses/by/4.0/). 